\title{
Retos para la economía internacional en un mundo cambiante
}

\author{
MAURO GUILLÉN ${ }^{\text {a }}$, EMILIO ONTIVEROS ${ }^{\text {b }}$
}

a Universidad de Pennsylvania, Wharton School of Business, 212 Lauder-Fisher Hall, 256 South 37th Street, Philadelphia, PA 19104, Estados Unidos.E-mail: guillen@wharton.upenn.edu

${ }^{b}$ Universidad Autónoma de Madrid, Facultad de CC.EE., Campus de Cantoblanco, c/ Francisco Tomás y Valiente, 5, 28049 Madrid, España.E-mail: emilio.ontiveros@uam.es

\section{RESUMEN}

Este artículo analiza las grandes transformaciones que está experimentando la economía mundial. Aborda los aspectos tecnológicos, demográficos, macroeconómicos, climáticos y de distribución de la renta, así como sus implicaciones sobre los nuevos equilibrios de poder en la economía internacional.

Palabras clave: Economía internacional, macroeconomía, cambio climático, demografía, tecnología.

\section{Challenges for the Global Economy in a Changing World}

\begin{abstract}
This paper explores the most important transformations that the global economy is going through. It focuses in aspects related to technological change, demographic dynamics, macroeconomic issues, climatic challenges and developments in the distribution of income. It also analyses the implications of these changes for the new balance of power in the global economy.

Keywords: International Economy, Macroeconomics, Climate Change, Demography, Technology.
\end{abstract}

Clasificación JEL: F00, F02, F20, F50, F60

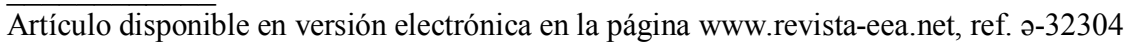




\section{INTRODUCCIÓN}

La economía mundial iniciaba el siglo XXI abordando una fase nueva en la dinámica de globalización, caracterizada por la confluencia de dos vectores de poderosa influencia en los años siguientes, tanto en la distribución de la actividad económica como en la distribución del poder geo-económico y geo-estratégico.

a) Los primeros compases de una nueva revolución tecnológica, de la mano de la rápida propagación de las TIC (Tecnologías de la Información y de la Comunicación). Afianzando su carácter de "tecnologías multipropósito" las empresas, las administraciones públicas, las entidades educativas y el ciudadano de a pie vieron multiplicarse sus posibilidades de acceso y procesamiento de la información y el conocimiento en un entorno de creciente interconexión y costes decrecientes. Pero esas tecnologías también provocaron un aumento de la tasa de creación destructiva en la economía y de la desigualdad social, en la medida en que el acceso a las mismas de la población no es homogéneo.

b) El ascenso de las economías emergentes cobrando un mayor peso en la economía global. Ya no solamente se trata de lugares donde se pueden realizar actividades productivas a bajo coste, sino también de mercados de consumo de clase media cada vez más amplios y de economías que exportan capital al resto del mundo, consecuentes con la acumulación de importantes superávits en sus balanzas de pagos.

Junto a estos factores, se mantienen algunas tendencias observadas desde años atrás. Entre ellas cabe destacar:

c) Una dinámica demográfica caracterizada por el progresivo envejecimiento de la población en las economías avanzadas y en algunas de las economías emergentes, sobre todo en China y Rusia.

d) Una creciente desigualdad en la distribución de la renta y de la riqueza, no tanto entre las naciones como en el seno de la mayoría de ellas, con el consiguiente deterioro del consumo y de la posición financiera de la clase media, y la proliferación de numerosos focos de tensión social de modo cada vez más frecuente.

e) Una asunción desigual de los imperativos transmitidos por la sostenibilidad del crecimiento entre los distintos países, estratos rurales y urbanos, y creciente dualismo dentro de las ciudades.

A los anteriores factores se sobrepuso a partir de 2008 el desencadenamiento de la crisis financiera y económica global, cuyas secuelas y efectos de diversa naturaleza trascienden a la no poco significativa erosión en el potencial de crecimiento de buen número de economías avanzadas, especialmente en la eurozona. Las secuelas de esa crisis, de las muy desiguales y controvertidas res- 
puestas de política económica y monetaria, no son pocas ni poco relevantes, especialmente en lo que se refiere al potencial de crecimiento, a la reducción del desempleo, a la capacidad para eludir esos riesgos de "estancamiento secular" que han renovado su vigencia propiciando analogías de este año 2014 con aquel de un siglo antes, 1914, que dio lugar a desenlaces desastrosos.

A la descripción de los anteriores aspectos están destinadas las notas que siguen. Son poco más que apuntes, sin una pretensión de gran exhaustividad, a modo de advertencias no por conocidas la mayoría de ellas menos relevantes. Estas necesariamente toman en consideración los trabajos que los autores llevan años realizando sobre la dinámica económica global, en sus clases en la Universidad Autónoma de Madrid, en la Escuela de Finanzas de Afi y en el Lauder Institute de Wharton, así como en diversas publicaciones, en particular su libro "Global Turning Points" y la versión en español actualizada, "Una Nueva época", de la que aparecieron diversos resúmenes en algunos medios de comunicación españoles y extranjeros.

\section{ENCUENTROS EN LA TERCERA FASE}

La coincidencia de la extensión de las tecnologías de la información y de la comunicación (TIC) y la adopción de ritmos de crecimiento superiores al promedio mundial en algunas economías consideradas menos desarrolladas son los dos episodios que, en nuestra opinión, mejor definen la entrada de la dilatada y discontinua dinámica de globalización en una nueva fase. Si la primera, ocupó el último tercio del siglo XIX hasta la emergencia de la primera guerra mundial, confiemos que la actual no se vea interrumpida por este aniversario del centenario con el inicio de esa fase de "estancamiento secular" que algunos autores han traído recientemente a colación como secuela más emblemática de la crisis económica todavía vigente. La segunda fase tendría su origen en la restauración del orden económico y financiero internacional pretendido con el sistema internacional creado en 1944 tras los acuerdos de Bretton Woods.

Del impacto de las TIC sobre diversos órdenes de la actividad económica y social existen evidencias suficientes ${ }^{1}$. Ya es amplia la verificación de la Ley de Moore acerca de la rápida capacidad de expansión de las posibilidades asociadas a esas tecnologías y su coexistencia con costes bajos o decrecientes. Como lo es la versatilidad de sus aplicaciones a distintos ámbitos de la actividad económica y social, y su fácil y barata permeabilidad geográfica.

La conectividad es el otro atributo esencial de esas tecnologías multipropósito que han cambiado la concepción de diversas áreas de la gestión empresarial o de la comunicación social y política. Sus consecuencias están lejos de haber sido completamente explicitas, más allá de las observaciones ya relevantes en la

\footnotetext{
${ }^{1}$ Puede verse a este respecto Ontiveros (2001).
} 
actividad económica, en la política o en los movimientos sociales. Así, por ejemplo, ámbitos como el de la ciberseguridad, son apenas una muestra de esa asimetría entre el ritmo de generación de innovaciones que estas tecnologías están posibilitando y el retraso de la capacidad de supervisión de las mismas.

El otro rasgo que concede singularidad a esta nueva fase es precisamente el encuentro de la amplia capacidad de acceso a esas tecnologías con la entrada en la escena económica global de un número creciente de economías no consideradas desarrolladas desde el punto de vista institucional, pero que acabarán disponiendo de un peso específico notable, de un protagonismo indudablemente superior.

Han sido más de dos décadas de crecimiento económico superior al promedio mundial las que han determinado que las economías emergentes lleguen a representar la mitad de la actividad económica global. Al mismo tiempo, no han dejado de fortalecer su posición financiera, reduciendo su vulnerabilidad frente a episodios de crisis financieras. Uno de los exponentes más representativos al respecto es el excedente en la cuenta corriente de la balanza de pagos de la mayoría de ellas, la reducción del endeudamiento externo y la consecuente acumulación de un importante stock de reservas internacionales de divisas. De hecho, un 75 por ciento de las reservas de divisas del mundo se encuentran ya en manos de las economías emergentes, con China a la cabeza.

Estrechamente vinculado a esa presencia creciente y resistente en la escena global es la presencia de empresas multinacionales provenientes de mercados emergentes, inmersas en una rápida expansión global ${ }^{2}$. La importancia de las mismas no puede minimizarse: De ser un fenómeno marginal han pasado a representar en 2010 el $25 \%$ de las 500 compañías más grandes del mundo, el $29 \%$ del número total de empresas multinacionales y el origen del $41 \%$ de los flujos de inversión directa extranjera de los últimos cinco años. La paradoja es destacada: su creciente poder y competitividad no se basa, necesariamente, en la posesión de tecnología o de aptitudes para la comercialización. Han reescrito las reglas de la competencia empresarial principalmente en provecho propio y a costa de las empresas multinacionales tradicionales de la OCDE.

\section{ALTERACIONES EN LOS EQUILIBRIOS QUE DEFINEN EL PODER A ESCALA GLOBAL}

La más explícita paradoja de las alteraciones en la distribución del poder global la presenta la reducción de significación global del G7. Este grupo originalmente constituido por las mayores economías del mundo (EEUU, Japón, Alemania, Reino Unido, Francia e Italia), aun viéndose obligado a dar cabida por razones esencialmente políticas a Rusia, no es representativo de las hege-

\footnotetext{
${ }^{2}$ En Guillén y Canal ( 2011) se abordan los rasgos de la emergencia de esas multinacionales.
} 
monías económicas en el mundo de hoy. Así se puso de manifiesto en la renovada vigencia que asumió el G20, comprensivo también de las más relevantes economías emergentes, con ocasión de la búsqueda de soluciones a la actual crisis económica y financiera.

Es verdad que EEUU sigue siendo la economía de mayor dimensión del mundo, con un PIB aproximadamente de 17 billones de dólares. Pero China aparece inmediatamente con uno superior a 9 billones de dólares, si en la valoración se emplean tipos de cambio de mercado. La diferencia en población y el dinamismo como operador en el comercio y la financiación global amparan esa anticipación de liderazgo global por tamaño e influencia geopolítica. Su población, 1.340 millones de habitantes en 2011, frente a los 312 millones de EEUU, ayuda también a explicar la distancia que todavía existe en términos de PIB por habitante: en 2011 el de China no superaba el 11\% del correspondiente a EEUU.

Esa posición de China como gran potencia global se afianza por su liderazgo en el comercio internacional. En 2013 el valor de sus exportaciones totales superó a las de EEUU en un 14\%, aunque sus importaciones fueron un 30\% inferiores, reflejando el todavía significativamente menor tamaño de su mercado. China ya es la mayor potencia comercial mundial, teniendo en cuenta tanto bienes como servicios.

La reciente actualización del International Comparison Programme (ICP) del Banco Mundial refleja el gran avance de la economía china en la clasificación por dimensión, hasta el punto de poder llegar a convertirse en la mayor al final de este año, cuando se utiliza como criterio tipos de cambio basados en la paridad del poder de compra (PPP, Purchasing Power Parities), en lugar de los tipos de cambio corrientes, que no reflejan el verdadero valor de la moneda china. La estimación anterior, de 2005, estimaba que sería en 2019 cuando China superaría a EEUU. Una revisión al alza es también la que tiene lugar en otras economías asiáticas como Indonesia.

Con independencia de la controversia metodológica que esa estimación ha generado $^{3}$ es un hecho que el poder económico convencional está hoy menos concentrado que hace apenas unos años. Una situación que contrasta con la fase en la dinámica de globalización que se inicia tras los acuerdos de Bretton Woods ${ }^{4}$, en la que la hegemonía estadounidense era manifiesta tanto en términos económicos como en la disposición de reservas internacionales. Con todo, según la renta per capita el liderazgo seguirá siendo de EEUU durante las próximas décadas.

\footnotetext{
${ }^{3}$ Véase Frankel (2014).

${ }^{4}$ En Ontiveros (1997) se describe la significación de esos acuerdos y su contraste con el basado en el patrón oro, vigente durante la primera fase de la globalización.
} 
En su dimensión estrictamente financiera EEUU seguirá manteniendo el liderazgo global, incluso tras el impacto de la crisis actual, originada en el seno de su sistema financiero. Su moneda apenas se ha visto contestada en su carácter vehicular y como moneda de reserva, incluso después de quince años de existencia del euro, con lo que ello significa de beneficios para su propia economía doméstica, en términos de posibilidades de financiación y de la posibilidad de definición de desequilibrios exteriores, como ha sido el caso en las tres últimas décadas. El desarrollo y sofisticación de su sistema financiero, la experiencia y calidad técnica de su banco central, también le siguen concediendo un protagonismo diferencial. Todo ello a pesar de la dependencia del ahorro exterior, de forma destacada del proveniente de las economías emergentes, y en especial de China. Las tenencias de reservas internacionales de divisas vuelven a ser expresivas al respecto: los 4.000 millones de dólares mantenidos al término del primer trimestre de 2014 por China dan cuenta de la capacidad inversora de esa economía y de su creciente influencia en las finanzas globales.

Uno de los principales retos que la economía de China ha de abordar de forma preferente es su modernización institucional, especialmente en aquellos ámbitos más cercanos a su apertura financiera al exterior. La convertibilidad exterior de su moneda, la liberalización de los flujos de capital, y la modernización de su sistema financiero son prioritarias. Dentro de estas, su sistema de supervisión de los operadores financieros puede todavía aportar algún disgusto que ralentice su necesaria homologación con las economías más avanzadas. Además, China carece de las instituciones legales necesarias para convencer a los inversores de que el país es seguro.

Donde las diferencias de China con las economías más avanzadas seguirá siendo importante es en la disposición de tecnologías, con un número significativo de empresas líderes en EEUU en todos los ámbitos, incluidos los más próximos a la industria de defensa y al correspondiente reflejo en el poder militar. China, sin embargo, tardará varias décadas en tener unas fuerzas armadas con la capacidad de proyectar fuerza regionalmente, y aún más si se trata de influir a lo largo y ancho del mundo.

En esa pugna por posiciones dominantes en la escena global el papel de Europa no ha registrado precisamente un avance. El conjunto de sus economías ha mantenido un ritmo de crecimiento más tibio, especialmente durante la crisis. Al mismo tiempo, su influencia geopolítica no ha ido paralela al ritmo de ampliación de la propia Unión Europea (UE). Ello ha sido el resultado, además de políticas poco orientadas al crecimiento y al fortalecimiento de los factores que impulsan la competitividad, a la ausencia de avances significativos en el fortalecimiento de la dinámica de integración. El conjunto de la UE, en especial la eurozona, se presenta hoy en la escena global como un bloque convaleciente, 
con serias dificultades en sus procesos decisionales y limitaciones competitivas de alcance.

La eurozona, efectivamente, ha acentuado durante esta crisis su declive relativo. El área monetaria tiene hoy un PIB de 16.7 billones de dólares. Su potencial de crecimiento se ha visto muy erosionado con el impacto diferencial de la crisis actual, tanto en términos de daños en la economía real, en los sistemas financieros e, incluso, en la erosión de las propias instituciones. Y la descapitalización sufrida no favorecerá su futuro dinamismo. Mantendrá excedentes en su cuenta corriente no muy inferiores a los de China en los próximos años, superiores al 2\% del PIB, pero seguirá lastrada por la insuficiencia de avances en el fortalecimiento de su dinámica de integración. Por la ausencia, en definitiva, de instituciones expresivas de un verdadera unión política.

\section{DINÁMICA DEMOGRÁFICA}

Por primera vez en la historia Japón y varios países europeos occidentales han invertido sus pirámides de población por edades y tienen más personas con más de 60 años que de menos de 20. También por primera vez, viven más personas en ciudades que en el campo y las que padecen de obesidad superan a las que pasan hambre. Según datos publicados recientemente en la revista The Lancet, el sobrepeso afecta a 2.100 millones de personas, casi un tercio de la población mundial; una tendencia ininterrumpida desde 1980.

La disminución del número medio de niños nacidos de cada mujer ha sido mucho más rápida en Europa, Asia Oriental (especialmente China y Japón) y, en menor medida, Norteamérica. Las mujeres de estas regiones disponen ahora de oportunidades económicas, políticas y sociales mucho mejores que en el pasado. Pero estas sociedades están envejeciendo muy rápidamente. En la primera década del siglo XXI, el mundo se ha hecho predominantemente urbano. Por primera vez en la historia hay más personas que viven en las ciudades que en el campo. La competencia por los recursos naturales ahora afecta no sólo a los minerales escasos, sino también a los alimentos y al agua.

La caída de la natalidad es una realidad a nivel global. Mientras que a mediados del siglo XX era 5 el número medio de hijos por mujer, en los albores del siglo XXI la proporción había caído a 2,5. En los países más desarrollados se produjo un acusado descenso inmediatamente después de la Segunda Guerra Mundial para situarse por debajo de 2 en los años setenta, mientras que en los países en vías de desarrollo se ha mantenido por encima de 4. La caída de la natalidad tiene sus causas principales en el aumento del acceso de las mujeres a la educación, que multiplica sus oportunidades laborales y les conduce a posponer los embarazos mientras se encuentran escolarizadas. Evidentemente, la mayor disponibilidad y efectividad de los métodos anticonceptivos también ha jugado un papel esencial, así como el proceso de urbanización. 
Ese descenso de la natalidad ha venido acompañado de un aumento generalizado de la esperanza de vida. En los países ricos vivimos hoy en día 15 años más por término medio que nuestros abuelos, hasta casi los 80 años. El aumento ha sido aún más vertiginoso en los países en vías de desarrollo, donde la esperanza de vida ha aumentado desde los 37 años en 1950 hasta los 60 en la actualidad. Las proyecciones indican una continuación de estos aumentos.

El hecho de que la natalidad haya caído rápidamente en los países más ricos y en algunas economías emergentes como China y Rusia viene generando una redistribución radical de la población en el mundo. Mientras que en 1950 África solamente tenía el 9 por ciento de la población mundial, en la actualidad alcanza ya el 15 por ciento, y Naciones Unidas estima que para el año 2100 podría llegar al 38 por ciento. Nigeria, el país más poblado, superará los 700 millones de habitantes antes de final de siglo. Países como India, Paquistán y Bangladesh también van a aumentar su población en términos absolutos y relativos. India será el país con una mayor población en una década, relegando a China a un segundo lugar. Mientras tanto, Europa verá su población decrecer tanto en términos absolutos como relativos, desde el 22 por ciento en 1950 hasta el 10 por ciento actual, y apenas un 6 por ciento en el año 2100. La otra parte del mundo con un acusado declive demográfico relativo es el Este de Asia, incluyendo China, Japón y Corea del Sur.

La caída de la natalidad y la mayor esperanza de vida conllevan un cambio muy rápido en la estructura de edad de la población. El envejecimiento de las sociedades europeas es un tema suficientemente conocido. Pero este proceso también está avanzando rápidamente en China, el sur de India y Brasil. Caminamos hacia un mundo en el que hay más abuelos que nietos. Además, la riqueza se concentra cada vez más en los grupos de edad más avanzada, en los que predominan las mujeres por su mayor longevidad y por su mayor incorporación al mercado de trabajo. Por tanto, el número de mujeres millonarias en el mundo -aquéllas con al menos un millón de dólares de patrimonio- van en aumento, y llega ya al 25 por ciento del total de millonarios en el mundo. Sin duda alguna, los patrones de consumo, ahorro e inversión de los millonarios en el mundo cambiarán debido al mayor número de mujeres con recursos financieros. Es importante recordar que las mujeres suelen definir una mayor aversión al riesgo que los varones en el manejo de las finanzas, y que suelen conceder más importancia a la educación y la salud.

El envejecimiento de la población en los países desarrollados pone en jaque al estado del bienestar, proyectando dudas sobre las promesas de pensiones y prestaciones sanitarias en sistemas en los que las prestaciones presentes se financian con los impuestos de los estratos de edad más joven que se encuentran empleados. Las corrientes migratorias podrían amortiguar parte de este efecto, si bien coincide que se viene produciendo un aumento de la hostilidad hacia los 
inmigrantes tanto en Europa como en Estados Unidos. El auge de los partidos políticos xenófobos es una manifestación palpable y preocupante de esta dinámica.

El crecimiento de las ciudades es otro proceso de cambio fundamental en el mundo actual. Cada semana se suman un millón y medio al total de habitantes urbanos en el mundo. Desde el año 2007, por primera vez en la historia de la humanidad, tenemos más personas viviendo en las ciudades que en el campo. En 1960 había solamente dos ciudades con más de 10 millones de habitantes, Nueva York y Tokio. En la actualidad son ya 21, y para el año 2025 la ONU estima que serán 35, la mayoría en India y China.

El crecimiento de las ciudades tiene dos consecuencias importantísimas. La primera es la necesidad de construir infraestructuras de todo tipo para acomodar la afluencia de personas, y sobre todo de aquéllas dirigidas a suministrar agua y alimentos. La segunda es la generación de nuevas pautas de desigualdad social tanto entre el campo y la ciudad como dentro de las ciudades, que cada vez son más duales. La pobreza urbana ha crecido considerablemente en las dos últimas décadas. Conviene recordar también que el cambio global afectará de manera drástica a los pobres urbanos en África y en el sur y este de Asia.

En definitiva, los cambios demográficos van a transformar el equilibrio geopolítico mundial, generando numerosas tensiones y una presión generalizada sobre los recursos naturales, especialmente el agua y los alimentos. Son muchos los expertos que vaticinan numerosos nuevos conflictos a causa de estos procesos de cambio tan acelerados.

\section{UN MUNDO CADA VEZ MÁS DESIGUAL}

Aunque la desigualdad en la distribución de la renta entre los países ha disminuido desde el comienzo del siglo XXI debido a la irrupción de las economías de mercado emergentes, la desigualdad dentro de los países ha continuado aumentando, planteando difíciles problemas políticos y sociales en los países desarrollados y en los considerados en vías de desarrollo. No deja de ser paradójico que la pobreza esté reduciéndose incluso en países en los que la desigualdad está aumentando.

La crisis desencadenada en 2007 ha contribuido a sensibilizar de forma creciente pero es verdad que no hace falta verificar las consecuencias de aquella sobre la distribución de la renta y de la riqueza para concluir en las amenazas que esa desigualdad constituye sobre la propia sostenibilidad del crecimiento económico. Desde la OCDE hasta el economista francés Thomas Piketty, autor de una obra ambiciosa y contundente en sus conclusiones, han difundido trabajos reveladores de la creciente concentración de la renta y de la riqueza a lo largo de los siglos. 
Más allá de consideraciones no poco relevantes como la dimensión moral de la desigualdad, lo más relevante en el contexto de estas notas es la amenaza al crecimiento que esa desigualdad supone. Las evidencias tan contundentes han hecho que la atención a este problema, su consideración como relevante para el futuro, deje a un lado las simplificaciones y etiquetamientos a quiénes advirtieron hace años de los efectos de esa tendencia.

La movilidad social es ciertamente reducida desde hace varias décadas en economías avanzadas, como la estadounidense y también, aunque en menor medida, las europeas, revelando que las ventajas sociales de la actual fase de globalización e incluso desde la anterior, no son homogéneas por grupos de población.

Las distintas sociedades toleran de manera muy distinta niveles elevados de desigualdad en la distribución de la renta y de la riqueza. Ello se debe a tradiciones culturales y políticas que afectan a los valores individuales y colectivos. Pero también conviene recordar que existe una relación inquietante entre la desigualdad y los incentivos para la inversión en capital humano y el trabajo. A niveles muy bajos de desigualdad - es decir, cuando la renta y la riqueza están distribuidas con casi completa equidad - los incentivos para invertir en capital humano son escasos. En el otro extremo, cuando la desigualdad es muy elevada tampoco se dan los incentivos para que la gente invierta en capital humano o se esmere en el trabajo. Por tanto, es imprescindible evitar grados elevados de desigualdad desde un punto de vista estrictamente económico.

Además, la desigualdad tiende a reducir el tamaño de la clase media. Se trata de un estrato social fundamental desde el punto de vista de la estabilidad sociopolítica y del mercado de consumo, dado que el grueso de la economía depende hoy en día del consumo. En la actualidad todavía hay un mayor número de personas en el mundo por debajo del umbral de la pobreza que los que resultan clasificados como clase media o ricos. Pero las proyecciones de la OCDE indican que aproximadamente en el año 2022 la clase media será el estrato social más numeroso en el mundo por primera vez en la historia. Esta tendencia viene impulsada por el crecimiento de las economías emergentes, donde cientos de millones de personas han pasado de ser pobres a convertirse en miembros de la clase media. Se estima que el mercado mundial de consumo de clase media asciende en la actualidad a 21,3 billones de dólares. En el año 2030 se calcula que podría alcanzar los 55,7 billones de dólares. La mayor parte de ese aumento se verificará en Asia, y una pequeña parte en Europa del Este.

En Estados Unidos y en Europa Occidental la clase media se encuentra en recesión debido al aumento de la desigualdad. En China la clase media sube en importancia gracias al crecimiento económico, pero no tan rápidamente como en India, donde la desigualdad social no está creciendo tan rápidamente. Mientras que en la actualidad más de la mitad del mercado global de consumo de 
clase media se encuentra en Europa y Estados Unidos, en el año 2040 serán India y China quienes tendrán los mayores mercados de consumo del mundo.

\section{LA SOSTENIBILIDAD COMO PRIORIDAD FUNDAMENTAL}

Existen pocas tenencias que además de ser asumidas de forma casi unánime generen tanta inquietud. El cambio climático, las amenazas a la propia sostenibilidad del crecimiento económico y de la extensión del bienestar, es una de ellas.

Los mejores científicos predicen que, sin una acción correctora, el cambio climático llegará a ser irreversible en algún momento crítico durante este siglo. Hacia el año 2030, los precios de los alimentos podrían duplicarse comparados con los de 2012 y la mitad de la población mundial podría verse afectada por una grave escasez de agua. El crecimiento en las economías emergentes ha ido acompañado de degradación medioambiental. En los países tanto desarrollados como en vías de desarrollo se pretende la búsqueda de la sostenibilidad. El énfasis está no sólo en la energía, sino también en la producción y el consumo ecológicos de bienes y servicios. La agricultura, la construcción y el turismo se han convertido también en objetivos de estos esfuerzos para alcanzar la sostenibilidad. La tecnología y el cambio de comportamiento de las personas se presentan como las únicas y complementarias soluciones.

La experiencia en otras crisis ha dejado como lección fundamental que es mejor prevenir que curar. Que no es necesario esperar a verificar los efectos del desastre antes de adoptar decisiones. De igual forma que la crisis todavía vigente ha contribuido a que los modelos macroeconómicos tomen en consideración la inestabilidad financiera, también habrán de hacer lo propio con las restricciones medioambientales, con la preservación del "capital natural", en definitiva.

\section{SECUELAS DE LA CRISIS ECONÓMICA Y FINANCIERA}

En la generación de la crisis desencadenada en el verano de 2007 en un segmento del mercado hipotecario del sistema financiero estadounidense, la acumulación de desequilibrios en algunas economías avanzadas desempeñó un papel importante. Con la excepción de Alemania y Japón presentaban abultados déficits exteriores, mientras que la mayoría de las economías emergentes mantenían superávits. El impacto adverso ha estado estrechamente relacionado con esas posiciones de endeudamiento externo de las economías. Ese impacto diferencial también se explica por la desigual celeridad y acierto de la reacción de las políticas económicas: más adecuado en el epicentro de la crisis, EEUU, que en la eurozona, donde se impusieron decisiones basadas en una austeridad que 
buscaba más los propósitos de enmienda de los deudores que la rápida recuperación de las economías. Las consecuencias de la crisis, especialmente en las economías de la eurozona consideradas periféricas, tardarán en superarse.

La crisis ha acentuado la inseguridad. Será necesario reforzar mecanismos precautorios, y disponer de una supervisión prudencial mucho más estricta. Pero la inseguridad se ha extendido al propio ámbito del análisis económico convencional, a su capacidad no solo para anticipar crisis como la actual, sino para ser capaz de deducir lecciones de la observación del mundo real y, en última instancia, para articular respuestas a las crisis.

Sin menoscabo de esas limitaciones analíticas, es igualmente cierto que no todas las decisiones de política económica han estado basadas en los propios fundamentos conceptuales del análisis económico, sino en consideraciones más referidas a la moral o al mero escarmiento. Es el caso del dominio de la austeridad fiscal como paradigma terapéutico, especialmente en la periferia de la eurozona. Los resultados son contundentes al respecto. Algo más de cuatro años de iniciada la aplicación de severas medidas de ajuste presupuestario las economías que lo han sufrido crecen poco y han elevado de forma muy significativa sus volúmenes de deuda pública. Lo han hecho por los desplomes en la recaudación tributaria, pero también por la exigencia de acudir en apoyo de los sistemas bancarios con recursos de los contribuyentes. Retrasos e insuficiencias de la política monetaria ayudan a entender igualmente el impacto diferencial de esa crisis sobre el conjunto de la eurozona.

Están en lo cierto, por tanto, quienes no asignan a las limitaciones del análisis esas respuestas políticas, sino justo lo contrario: han sido las decisiones las que han tratado de enmendar el sentido común económico, forzando presunciones como la naturaleza expansiva de la austeridad o la existencia de determinados umbrales de deuda pública a partir de los cuales el crecimiento de las economías se tornaba negativo. Ello ha hecho que con los resultados tan adversos observados en la eurozona, también haya aumentado el grado de escepticismo acerca de la capacidad del análisis económico y de la utilidad los economistas.

\section{PANORAMA POLÍTICO EN MUCHAS PARTES DEL MUNDO HA DADO UN VUELCO HACIA LA INCERTIDUMBRE Y EL CAOS}

La buena noticia desde el punto de vista geopolítico es que el número de democracias es mucho mayor que el de dictaduras, tendencia que comenzó tras el fin de la Guerra Fría. Pero también hay malas noticias, en primer lugar son ya más de 50 los países que, pese a ser formalmente democráticos, tienen líderes que se comportan de forma autocrática, como en los casos de Rusia o Venezuela. En segundo lugar, existen al menos 40 países que son estados fallidos, es 
decir, situaciones en las que la autoridad del estado está en entredicho hasta el punto de que hay anarquía o caos, como en el caso paradigmático de Somalia. Esta tendencia ha causado importantes problemas en la economía y el comercio mundiales y convertido el terrorismo en la forma dominante de conflicto violento, mientras que en el siglo XX lo eran las guerras. Además, conviene recordar que la geografía de la inestabilidad geopolítica (África, Oriente Próximo, y sudeste asiático) coincide con una gran concentración de pobreza, corrupción, crecimiento demográfico y riqueza en recursos naturales y energía. El futuro de la economía global depende en gran medida de la resolución de estos problemas.

Conflictos y tensiones globales requieren soluciones y plataformas institucionales verdaderamente representativas de esas alteraciones que se han producido en la escena económica y geopolítica global. De su rápida concreción dependerá el alejamiento definitivo del espectro de una larga fase de bajo crecimiento económico y aumento de las tensiones geopolíticas. Queda también por resolver el nuevo equilibrio de poderes en el mundo en general, y en los organismos internacionales en particular, entre las economías emergentes y las economías desarrolladas. El mundo del siglo XXI ya es muy distinto al de la segunda mitad del siglo anterior, y seguirá cambiando constantemente en las próximas décadas.

\section{REFERENCIAS BIBLIOGRÁFICAS}

FRANKEL, J. (2014). China is not yet number one. Vox, 9 de mayo.

GUILLÉN, M. y CANAL, E. (2011). Las Nuevas Multinacionales. Madrid: Ariel.

GUILLÉN, M. y ONTIVEROS, E. (2012). Global Turning Points. Understanding the challenges for business in the 21st. Century. Cambridge University Press.

ONTIVEROS, E. (1997). Sin orden ni concierto. Medio siglo de relaciones monetarias internacionales. Madrid: Escuela de Finanzas Aplicadas, Afi.

ONTIVEROS, E. (2001). La economía en la red. Nueva economía, nuevas finanzas. Madrid: Taurus.

ONTIVEROS, E. y GUILLÉN, M. (2012). Una Nueva Época. Los grandes retos del siglo $X X I$. Madrid: Galaxia Gutenberg. 
